\title{
Using the CAI software (InventionTool 3.0) to solve complexity problem
}

\author{
Zhang Peng, Tan Runhua \\ School of Mechanical Engineering, Hebei University of technology , \\ Tianjin, 300130, People's Republic of china, \\ landi1979@sina.com, rhtan@hebut.edu.cn
}

\begin{abstract}
InventionTool 3.0, which is a CAI software based on TRIZ, is created by Institute of Innovation of Hebei University of Technology. Using the CAI software, designers can solve innovation problem. During the design process, the InventionTools 3.0 plays a very important role. Firstly, the technical evolution module of the InventionTools 3.0, as an effective method, is used to obtained conceptual design and several solutions. Secondly, using the Complexity Theory based on the Axiomatic Design, the solutions are analyzed. Finally, according to analysis result, the best solution would be obtained. In this paper, the pill counting system is used as an complexity problem to show how the InventionTool 3.0 and the Complexity Theory based on the Axiomatic Design is applied.
\end{abstract}

\section{Introduction}

The dropping pill is a Chinese traditional medicine and has won the favor of people all over the world. For the dropping pill is quite unique, some existing production line is unable to satisfy the requirements of its production. A new production line is needed, which includes three sections: the dropping pill machine, the scratching pill machine and the packaging machine. For reducing the cost and improving the competition, we pay attention to increasing the quality of design. Using the InventionTools 3.0 is an important way to achieve it. During the design process, the InventionTools 3.0 plays a very important role. Firstly, the technical evolution module of the InventionTools 3.0 , as an effective method, is used to obtained conceptual design and several solutions. Secondly, using the Complexity Theory based on the Axiomatic Design [1], the solutions are analyzed. Finally, according to analysis result, the best solution would be obtained.

The pill counting system, which has shown the application of the law and routes of system evolution, is a sub-system in the production line of dropping pill. During

Please use the following format when citing this chapter:

Peng, Z., Runhua, T., 2007, in IFIP International Federation for Information Processing, Volume 250, Trends in Computer Aided Innovation, ed. León-Rovira, N., (Boston: Springer), pp. 115-123. 
the design process, the InventionTools 3.0 is used. What is the InventionTools 3.0 ? How did we use the InventionTools 3.0? Why do we apply the Complexity Theory based on the Axiomatic Design?

\section{Preliminaries}

\subsection{The TRIZ technology forecasting}

The TRIZ technology forecasting [2] developed by Altshuller [3] is a set of patterns and paths, which show the trends of technological systems evolution in structures. These patterns and paths are revealed by analysis of hundreds of thousands of invention descriptions available in the world patent databases. The most important finding is that the patterns and paths revealed in one engineering field can be transferred to other kinds of artificial systems [4]. The law and routes of system evolution are described that the routes of system evolution are the order of structural evolution of the core technology. All in all, we can obtain the trends of technological systems by the TRIZ technology forecasting.

When we develop a new product, it is necessary for the function units to be described precisely and detailedly and for the system model to be established. The chief task for designers is to confirm the function units, analyze the correlation among the function units and establish the function model of system. In order to realize the demand mentioned above, function analysis is adopted. According to technology evolution, several solutions are obtained when Invention Tool 3.0 is used. If the function unit is independent, the best could be obtained in the several solutions. On the other hand, if the function unit is not independent, the best might be hidden in the several solutions. A system method is needed to obtain design optimization. The Complexity Theory based on the Axiomatic Design provides the theoretical basis for design optimization.

\subsection{Complexity Theory based on the Axiomatic Design}

In 1999 N.P. Suh put forth the Complexity Theory based on the Axiomatic Design method. What is complexity? How do we reduce complexity? Why do people think that a product with many parts is complex? Complexity is measured in the functional domain rather than in the physical domain, which distinguishes this theory from other complexity theories and yields a unique and inherent perspective on complexity [5].

The design effort may produce several designs, all of which may be acceptable in terms of the technology evolution. It is likely that different designers will come up with different designs, because there can be many designs that satisfy the function requirement [5]. However, one of these designs is likely to be superior to the others. The Complexity Theory based on the Axiomatic Design is useful in selecting the best among those designs that are acceptable. Among the designs that acceptable 
from the functional point of view, one may be superior to others in terms of achieving the design goals as expressed by the functional requirements. The Complexity Theory based on the Axiomatic Design states that the design with the highest probability of success is the best design.

Complexity is defined as a measure of uncertainty in achieving the functional requirements (FRS) of a system [6]. A design is called complex when its probability of success is low, that is, when the information content required to satisfy the FRs is high. A physically large system is not necessarily complex if the information content is low. Conversely, even a small system can be complex if the information content is high [6].

\subsection{InventionTool 3.0}

InventionTool 3.0, which is a CAI software based on TRIZ, is created by Institute of Innovation of Hebei University of Technology. Figure 1 shows the homepage of the InventionTool 3.0.

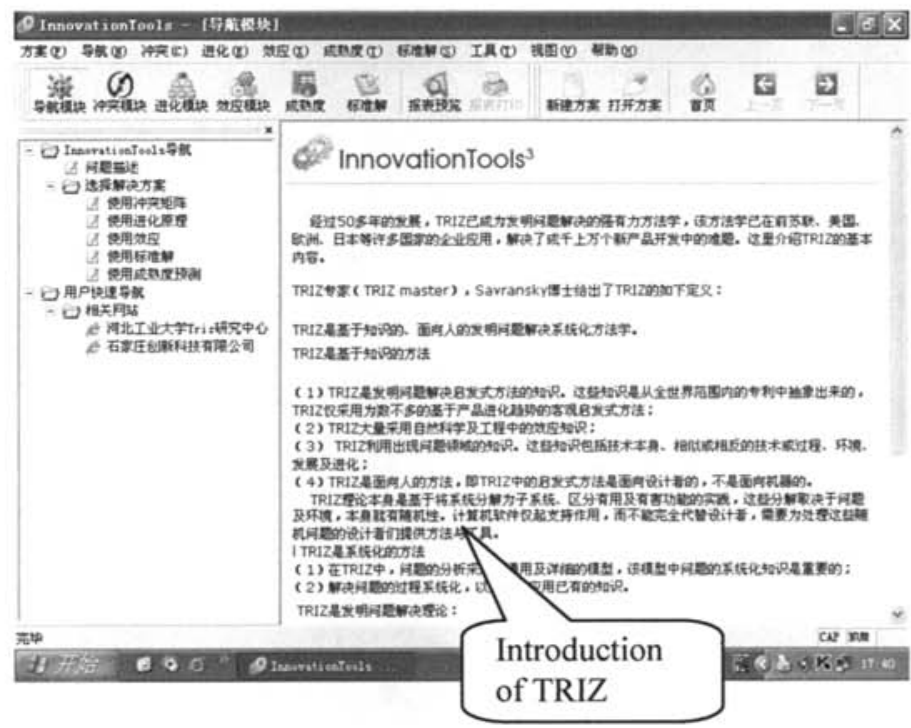

Fig.1. The homepage of the Invention Tool 3.0

At present, CAD software can be used in the technical design and the detailed design, but can not be used in the conceptual design. The CAD software can not be used in helping the designer to obtain innovation plans. Using the CAI software, designers can solve the "innovation problem".

There are three modules in the InventionTool 3.0: the evolution module, the conflict module and the effect module. According to these modules, designers can obtain several solutions. 
The InventionTool 3.0 solves "innovation question" based on TRIZ [3]. TRIZ was developed in Russia by Genrich Altshuller, a talented scientist and inventor, and his followers. Altshuller's work with TRIZ began in the 1940s and, to date, much experience in applying TRIZ application to various areas of human activity has been amassed [3]. TRIZ is based on the study and application of the patterns of evolution of various systems-technological machines, manufacturing processes, scientific theories, organizations, works of art, and so on. Based on these patterns, methods have been developed for searching for creative solutions. TRIZ includes several parts: TRIZ tools, repository and technology forecasting [4]. A person masters all of them is difficult. So, the computer aided innovation software is necessary. For cutting the designing time and improving the level of innovation, designers pay attention to using the computer aided innovation software. Figure 2 shows the homepage of the evolution module.

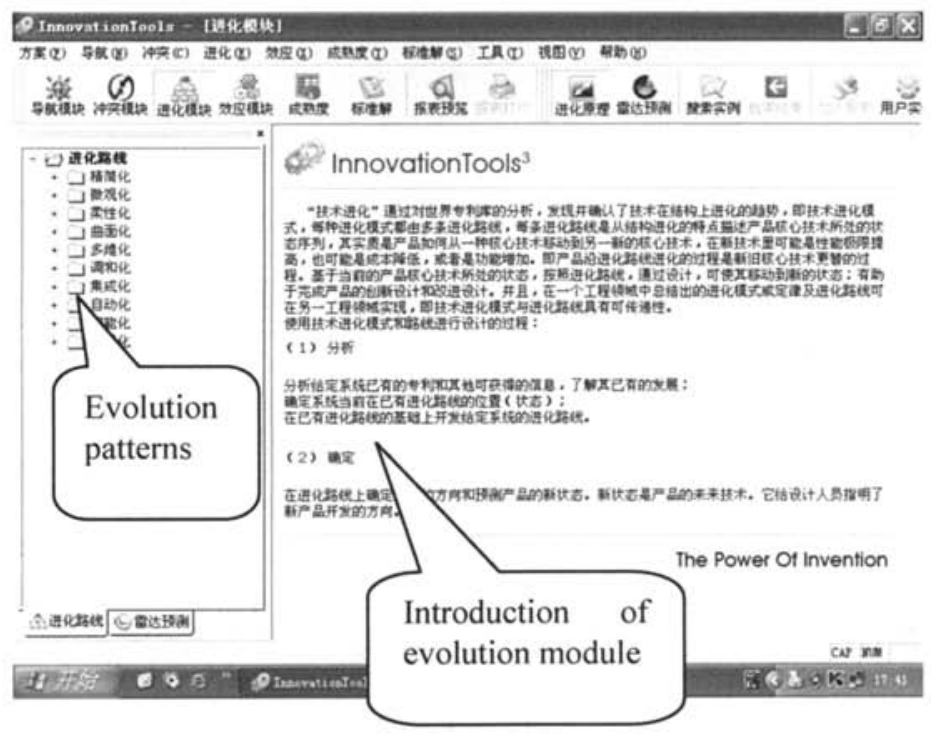

Fig.2. The homepage of evolution module.

\section{Case study}

The design process of the pill counting system is a typical example of the whole design process. In the process, InventionToo1 3.0 is used. The pill counting system is divided into two parts: the pill boards and counting sensors.

The principle of the pill counting system is: the sensor send out rays which can be reflected by pills. If the sensor doesn't receive the ray which is sent out by itself and reflected by the pill, indicate there is not a bill. Figure 3 shows the principle of the pill counting system. 


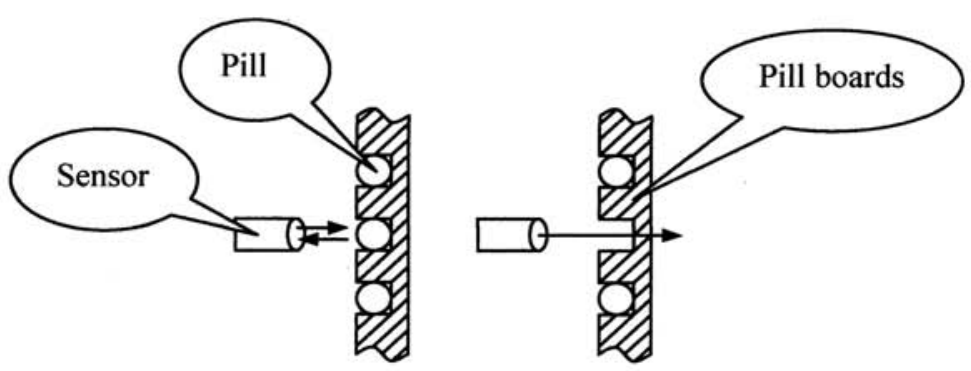

Fig.3. The principle of the pill counting system.

\subsection{Design the arrangement of the counting sensors}

According to the evolution path "Mono - bi - poly" [8] of the InventionTool 3.0, several solutions were obtained when the function requirement of the pill counting system was known. Figure 4 shows the evolution path "Mono - bi - poly" of the InventionTool 3.0.

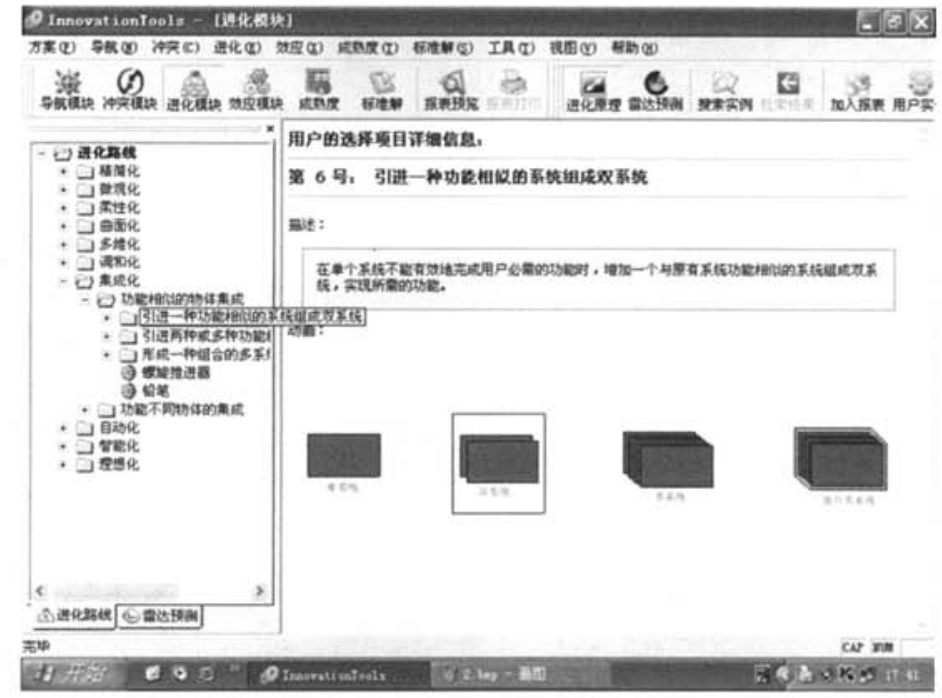

Fig.4. The evolution path "Mono - bi - poly"

Using the evolution path "Mono - bi - poly" of the InventionTool 3.0, the arrangement of the counting sensors can be designed as figure 5 . 


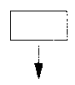

(a)

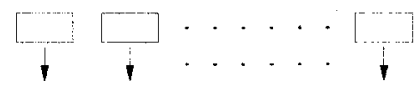

(b)

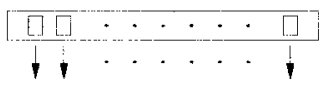

(c)

Fig.5. Three projects of the counting sensors.

Now, let's analyze the three projects using the complexity theory based on the Axiomatic Design method. When the project one is adopted, we will see a single system with only one sensor. In this project, the counting system's function can be achieved easily. But the project is inefficient, so it is not available. According to the evolution path" Mono - bi - poly", we can choose multi-system. Fig.6-b shows the project two. Several same sensors are used. The project two is more efficient than the project one. But the sensors' operational complexity may be increased. This project is not available, too. Is there a project which will increase efficiency but not complexity? Project three, which is shown in fig.6-c, can achieve it. The sensors are integrated. Efficiency is improved, and operational complexity is not increased.

We apply the three projects in counting system and analyze their feasibilities. We regard the cylinder as the pill board.

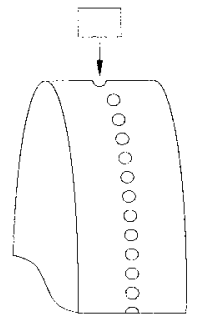

(a)

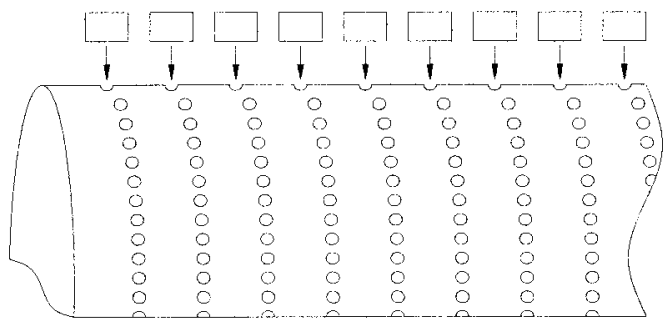

(b)

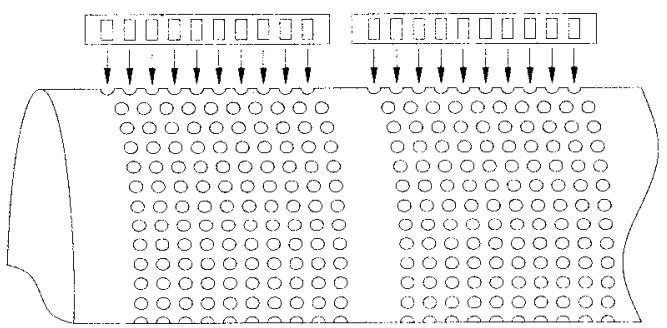

(c)

Fig.6. Three projects of the counting sensors

Fig.6-a shows the project one, there is only one set of holes are used to put pills. It is easy to design and manufacture this system. But this system is not available for its inefficient. 
Fig.6-b shows the project two, this system involves several sensors and several sets of holes. Although efficiency is improved, operational complexity is increased simultaneously. Besides, complexity of installation is also increased.

Fig.6-c shows the project three. For reducing the complexity and improving the efficiency, the sensors are integrated. Is the project three better than project two? By integrated, the size of the sensors will be reduced, and the space between two sets of pill holes will be reduced, too. Thus, the pill board's manufactural complexity may be increased.

\subsection{Design the pill boards}

To sum up, we need to complete the design of the pill boards to gain a satisfying counting system. We design the pill boards based on the evolution module of the InventionTool 3.0 correspond with the design of the sensor.

When we design the pill board, the following question must be considered. How to improve efficiency? Increasing the size of the cylinder is an important way to achieve it, but it will increase the complexity of the system. The evolution path "segmentation of objects and substances" [9] of the InventionTool 3.0, as an effective method, is used to obtain the solutions of the problems.

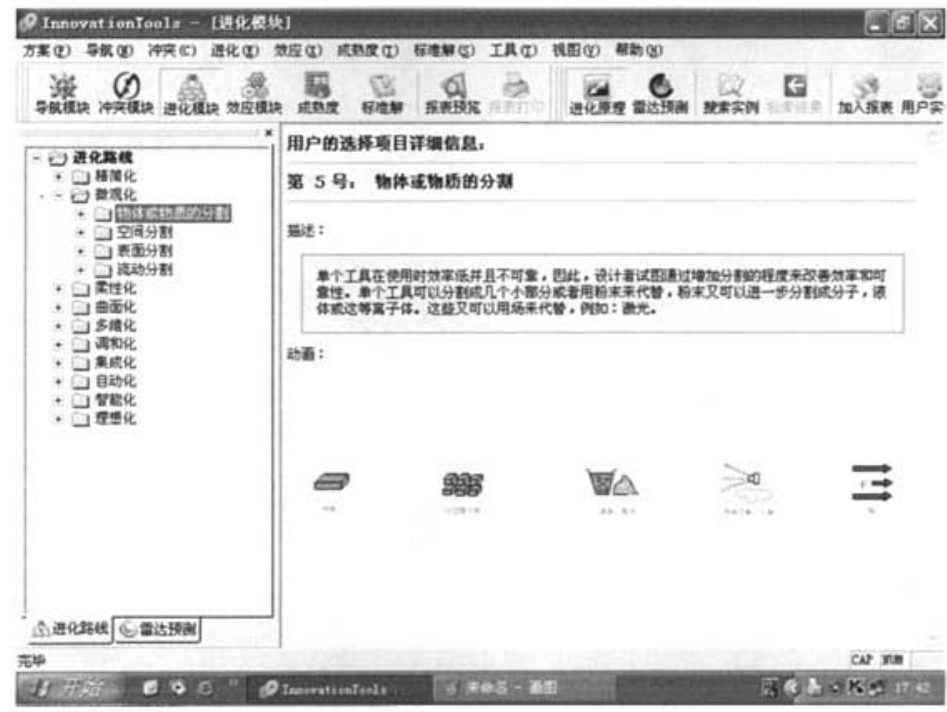

Fig.7. The evolution path "segmentation of objects and substances"

According to the evolution path "segmentation of objects and substances", the design has smaller information content when the cylinder is divided into several parts. That is to say, the system's complexity is reduced. The system in the project one only has one sensor and a set of pill hole, so its cylinder can't be divided into several parts. We analyze the project two and project three.

The cylinder in the project two is divided into several parts. It is shown in fig. 8 . 


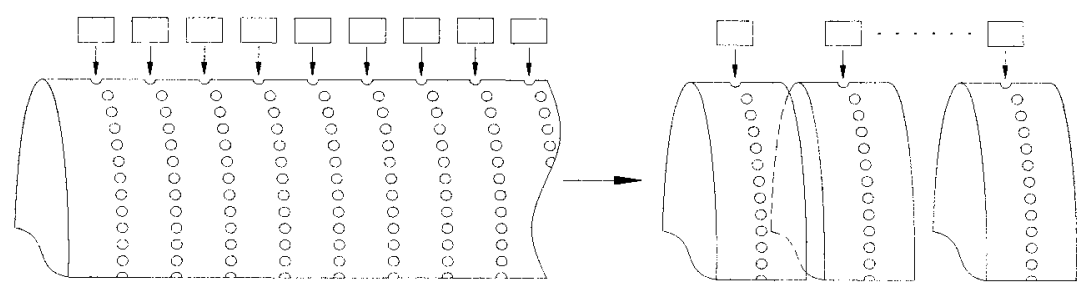

Fig.8. Improving the project two

The manufactural complexity is reduced, but many other complexities, such as installation complexity come forth with it. The complexity of the counting system is not improved evidently. The project two is not available.

As is shown in fig.9, the cylinder in the project three is divided into several parts. The manufactural complexity is reduced, and it's convenient to install. The project three is efficient with smaller complexity. The project three is the best design.

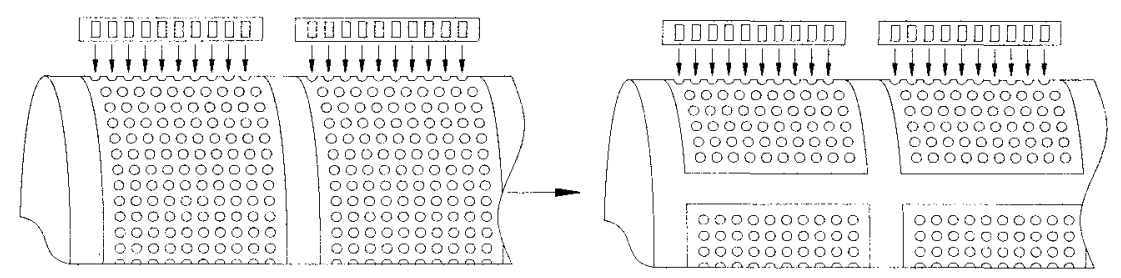

Fig.9. Improving the project three

\section{Conclusions}

The InventionTool 3.0 can be used to support designers to find several projects. The best is usually hidden and designers do not know it. According to the Complexity Theory based on the Axiomatic Design method, designers can find it and implement it in the design of products. Firstly, some projects are raised by the design of the pill counting sensor. Secondly, the projects are analyzed for their feasibilities based on the Complexity Theory. Lastly, the smallest information content project is obtained, so it is the best.

The CAI software plays a very important role in design. Designers pay attention to the computer aided innovation software. As the first domestic CAI software in china, InventionTool 3.0 has won the favor of national enginery company, and has been already used by several companies. As is shown in the process of designing the pill counting system, the Invention Tool 3.0 may provide many design projects for the designer, but the choice of the projects still depend on the designer oneself. The 
InventionTool 3.0 can't do it .The tendency of the Invention Tool $\mathrm{x}$ software is adding the optimization module based on the Complexity Theory.

\section{Acknowledgements}

The research is supported in part by the Chinese Natural Science Foundation under Grant Numbers 50675059 and Chinese national 863 planning project under Grant Number 2006AA04Z109. No part of this paper represents the views and opinions of any of the sponsors mentioned above.

\section{References}

1. 1 Suh N.P. Axiomatic Design: Advances and Applications (Oxford University Press, New York, 2001).

2 Suh N.P.The Principles of Design(Oxford University Press, New York, 1990).

3 Altshuller G, The Innovation Algorithm, TRIZ, systematic innovation and technical creativity, Technical Innovation Center, INC., Worcester, 1999

4 Savransky S D, Engineering of creativity, CRC Press, New York, 2000

5 Suh N P. "A theory of complexity, periodicity, and design axioms", Research in Engineering Design, Vol.11, 1999, pp. 116-131.

6 Suh N P. Design-in of Quality through Axiomatic Design. IEEE Transactions on Reliability, 44(2): 1995.

7 TAN Runhua. A macro process model for product innovation using TRIZ. The eighth world conference on integrated design \& process technology, Beijing, China, 2005, 766773

8 Twiss B., Managing technological innovation, Fourth edition, Pitman Publishing, London, 1992

9 Twiss B., Forecasting for technical decisions, Peter Peregrinus, London, 1992

10 Fey V.R. and Rivin E.I., Guided technology evolution (TRIZ Technology Forecasting), http://www.trizgroup.com/article2.htm 1

11 Zusman A., Zlotin B., Zainiev G., An application of directed evolution, $\mathrm{http}: /$ www.ideationtriz.com/Endoscopic_case_study.htm 